\title{
Interfering cellular lactate homeostasis overcomes Taxol resistance of breast cancer cells through the microRNA-124-mediated lactate transporter (MCT1) inhibition
}

\author{
Lu Hou, Yi Zhao*, Guo-qing Song, Ying-han Ma, Xiao-hu Jin, Si-li Jin, Yi-han Fang and Yi-chong Chen
}

\begin{abstract}
Background: Breast cancer, the most common invasive cancer of women, is a malignant neoplasm and the second main cause of cancer death. Resistance to paclitaxel (Taxol), one of the frequently used chemotherapy agents for breast cancer, presents a major clinical challenge. Recent studies revealed that metabolic alterations of cancer cells play important roles in chemo-resistance.

Materials and methods: In this study, Human breast cancer cells, BT474, SKBR3 and MCF7 were used to study the causal relationship between the lactate exporter, MCT1 (SLC16A1)-modulated glucose metabolism and Taxol resistance of breast cancer cells. Taxol resistant breast cancer cells were established. The intracellular lactate and extracellular lactate levels as well glucose uptake and oxygen consumption were measured. MicroRNA-124 expressions were detected by qRT-PCR from both breast cancer patient samples and breast cancer cells. Target of miR-124 was predicted and verified by Western blot and luciferase assay. An xenograft mice model was established and evaluated for the in vivo tumor therapeutic effects of MCT1 inhibitor plus microRNA-124 treatments.

Results: Low toxic Taxol treatments promoted cellular glucose metabolism and intracellular lactate accumulation with upregulated lactate dehydrogenase-A (LDHA) and MCT1 expressions. By establishing Taxol resistant breast cancer cell line, we found Taxol resistant cells exhibit upregulated LDHA and MCT1 expressions. Furthermore, glucose consumption, lactate production and intracellular ATP were elevated in Taxol resistant MCF7 cells compared with their parental cells. The miR-124, a tumor suppressive miRNA, was significantly downregulated in Taxol resistant cells. Luciferase assay and q-RT-PCR showed MCT1 is a direct target of miR-124 in both breast cancer cell lines and patient specimens. Moreover, co-treatment of breast cancer cells with either MCT1 inhibitor or miR-124 plus Taxol led to synergistically cytotoxic effects. Importantly, based on in vitro and in vivo results, inhibition of MCT1 significantly sensitized Taxol resistant cells. Finally, rescue experiments showed restoration of MCT1 in miR-124 overexpressing cells promoted Taxol resistance.
\end{abstract}

Conclusions: This study reveals a possible role of miRNA-214-mediated Taxol resistance, contributing to identify novel therapeutic targets against chemoresistant breast cancers.

Keywords: Breast cancer therapy, MicroRNA-124, Taxol resistance, Lactate homeostasis, MCT1 inhibitor

*Correspondence: zhaoy5@sj-hospital.org

The 1st Breast Surgical Department, Affiliated Shengjing Hospital of China

Medical University, Shenyang 110004, Liaoning, China 


\section{Background}

Breast cancer is one of the most frequently diagnosed cancers among women [1, 2]. In addition to surgery, adjuvant systemic therapies including endocrine therapy, anti-HER2 therapy, and chemotherapy have been reported to effectively reduce the risk of local and distant recurrence [3]. Taxol (paclitaxel) is an essential chemotherapeutic agent for the treatment of multiple types of tumor, including breast cancer through disrupting the dynamic equilibrium between tubulin dimers and their polymerized form [4]. Yet, a significant percentage of breast cancer patients fail to respond to initial chemotherapy due to development of resistance during the treatment with Taxol, limiting the clinical efficacy of Taxol $[4,5]$. Thus, it is imperative to investigate novel strategies which could overcome chemoresistance in cancers.

Warburg first postulated that cancer cells, in contrast to normal cells rely on glycolysis than oxidative phosphorylation to produce ATP for proliferation [6]. Moreover, in cancer cells, pyruvate produced from glycolysis was converted to lactate by lactate dehydrogenase A (LDHA) rather than acetyl-CoA [7]. This phenomenon is called the "Warburg effect" [8]. Dysregulated glycolytic flux leads to high levels of lactate, which is exported by transporters (MCTs) on the plasma membrane [9]. The MCT (monocarboxylic acid solute transporters) family includes 14 members, and MCT1-4 have been reported to mediate bi-directional transport of lactate [10]. Studies demonstrated that MCT1 expressions positively correlate with glycolytic metabolism and malignancy in breast cancer [11]. Moreover, MCT1 inhibition could suppress growth and induce cell death of breast cancers through disruption of glycolysis and glutathione synthesis [12], suggesting blocking the MCT1-mediated glycolysis might contribute to improvement of chemotherapeutic effects.

MicroRNAs (miRNAs) are a group of small, noncoding RNAs (22-25 nucleotides) that play important roles in diverse cancer processes, such as tumorigenesis, development, differentiation, invasion, migration and cell death [13, 14]. MiRNAs lead to post-transcriptional silencing of their target mRNAs through complementary binding to the $3^{\prime}$ untranslated region (UTR) of mRNA targets $[14,15]$. Multiple studies reveal that miR-124 negatively regulates carcinogenesis with the observations that miR124 expression level is significantly downregulated in oral squamous cell carcinoma (OSCC) [16], glioma [17], colon cancer [18], lung cancer [19], hepatocellular carcinoma (HCC) [20], breast cancer [12] and bladder cancer [21]. However, the molecular mechanisms underlying the miR-124-regulated malignant phenotype of breast cancer cells are still under investigation. In this study, roles of the axis miR-124-MCT1-Taxol sensitivity of breast cancer will be studied. We will predict and verify the direct target of miR-124 in breast cancer cells and patient specimens. Furthermore, the synergistic effects of the combination of MCT1 inhibitor and Taxol treatments on breast cancer will be investigated in vitro and in vivo.

\section{Materials and methods \\ Cell culture and tumor specimens}

Human breast cancer cell lines BT474, MCF7, SKBR3 and MDA-MB-231 were purchased from the American Type Culture Collection (Manassas, VA, USA). All cells were cultured in DMEM/F12 (Dulbecco's Modified Eagle Medium: Nutrient Mixture F-12) medium and maintained at $37^{\circ} \mathrm{C}$ in a $5 \% \mathrm{CO}_{2}$ humidified incubator. A cohort of 60 human primary breast cancer specimens during surgery, collected during the surgery at the Affiliated Shengjing Hospital of China Medical University, China, were examined in this study. All protocols concerning the use of patient specimens were approved by the Medical Ethics Committee of Affiliated Shengjing Hospital of China Medical University. The informed consent was signed by all the patients involved in the study. All breast cancer specimens were from female patients classified according to current World Health Organization criteria. The ages of adult patients were from 25 to 44 years.

\section{Antibodies and reagents}

Paclitaxel and SR13800 were purchased from SigmaAldrich (St. Louis, MO, USA). Antibodies against $\beta$-actin (\#4970) and LDHA (\#3582) were purchased from Cell Signaling (Beverly, MA, USA). Monoclonal mouse antibody against MCT1 was purchased from ThermoFisher Scientific (\#MA5-18288, Waltham, MA, USA). Control siRNA and MCT1 siRNA (5'-GAGGAUGAAUCACUA AGUA-3') were synthesized by Ribobio (Guangzhou, China).

\section{MiRNAs transfection and siRNA knockdown}

MiRNAs and siRNA transfections were performed using Lipofectamine RNAiMAX reagents (ThermoFisher Scientific, Waltham, MA, USA) according to the manufacturer's instructions. Briefly, $2 \times 10^{5}$ cells/well were plated in 6-well plate for overnight. After refreshing medium, cells were transfected with miRNAs $(25 \mathrm{nM})$ or siRNA $(50 \mathrm{nM})$ for $48 \mathrm{~h}$ followed by downstream experiments.

\section{Glucose consumption and lactate assays}

The glucose consumption and lactate product assays were performed as previously reported [22, 23] using the Glucose Uptake Assay Kit (Colorimetric) (ab136955) and L-Lactate Assay Kit (Colorimetric) (ab65331) from 
Abcam (Cambridge, UK) according to the manufacturer's instructions. The intracellular and extracellular lactate were detected using an absorbance-based assay kit (Biovision, Milpitas, CA, USA) according to the previous report [12]. All experiments were performed in triplicate and repeated three times.

\section{ATP/ADP ratio assay}

Intracellular ATP/ADP ratio was determined with an ADP/ATP Ratio Assay kit (Cat. No. ab65313, Abcam) according to the manufacture's instruction. Briefly, $1 \times 10^{4}$ cells/well were plated in 96 well plates for overnight. The luminescence was measured in a luminometer. ATP/ ADP ratio was calculated as $1-[$ Data $D-$ Data $C] /[$ Data B - Data A]. Data D: sample signal $\sim 2$ min after addition of $10 \mu \mathrm{L} 1 \times$ ADP Converting Enzyme to cells; Data C: sample signal prior addition of $1 \times$ ADP Converting Enzyme to cells; Data B: sample signal $\sim 2$ min after addition of cells to reaction mix; Data A: background signal of reaction mix. All experiments were performed in triplicate and repeated three times.

\section{Oxygen consumption rate (OCR)}

OCR was measured using the Seahorse Bioscience XF96 Analyzer according to previous reports [12]. Final concentration of agents used was oligomycin $1 \mathrm{mmol} / \mathrm{L}$ (Calbiochem), carbonyl cyanide 4-(trifluoromethoxy) phenylhydrazone (FCCP) $300 \mathrm{nmol} / \mathrm{L}$ (Sigma), and rotenone $100 \mathrm{nmol} / \mathrm{L}$ (Sigma). Protein concentration of each sample was measured using Bradford method to normalize OCR results. All experiments were performed in triplicate and repeated three times.

\section{Measurement of cell viability}

Cell viability was measured by MTT assay (MD Millipore, Billerica, MA, USA). Briefly, cells were cultured at $3 \times 10^{4}$ cells/well in a 96-well culture plates for overnight. Then 3-(4,5-dimethylthiazol-2-yl)-2,5-diphenyltetrazolium bromide (MTT) at a concentration of $5 \mathrm{mg} / \mathrm{mL}$ was added to each well at $10 \mu \mathrm{L} / 100 \mu \mathrm{L}$ followed by incubation at $37{ }^{\circ} \mathrm{C}$ for $2 \mathrm{~h}$ in a cell culture incubator. The medium was replaced with $100 \mu \mathrm{L}$ dimethyl sulfoxide (DMSO), and the absorbance for each well was measured at $570 \mathrm{~nm}$ using a scanning multiwell spectrometer (BioTek instruments, Inc., Burlington, VT, USA). All experiments were performed in triplicate and repeated three times.

\section{RNA isolation and real-time PCR}

Total RNA was extracted using a TRIzol Reagent (Invitrogen, Carlsbad, CA) according to the manufacturer's instruction and subsequently treated with RNase-free DNase I (Fermentas, San Diego, CA, USA). For detection of mRNA expressions, cDNA synthesis was performed from $1 \mu \mathrm{g}$ total RNA using a SuperScript First-Standard Synthesis system for RT-qPCR (Invitrogen Life Technologies). Real-time PCR was performed by an ABI PRISM 7900 Sequence Detection System (Applied Biosystems, Foster City, CA, USA) using Quanti-Tect SYBR Green PCR mixture (Qiagen, Hilden, Germany). The qPCR primers were: MCT1: forward: 5'-GTGGCTCAGCTC CGTATTGT-3, reverse: 5'-GAGCCGACCTAAAAG TGGTG-3'; LDHA: forward: $5^{\prime}$-TGGAGTGGAATGAAT GTTGC- $3^{\prime}$, reverse: $5^{\prime}$-ATAGCCCAGGATGTGTAG CC- $3^{\prime}$; and $\beta$-actin: forward: 5'-AGGCACCAGGGC GTGAT- $3^{\prime}$, reverse: $5^{\prime}$-GCCCACATAGGAATCCTT CTGAC- $3^{\prime}$. For detection of miRNAs, reverse transcription reaction was performed using the TaqMan Advanced miRNA cDNA Synthesis Kit (ThermoFisher Scientific, Waltham, MA, USA) according to the instructions. qPCR was performed by ABI PRISM 7900 Sequence Detection System. The reactions were incubated in a 96-well optical plate at $95{ }^{\circ} \mathrm{C}$ for $10 \mathrm{~min}$, followed by 40 cycles at $95{ }^{\circ} \mathrm{C}$ for $15 \mathrm{~s}$ and $60{ }^{\circ} \mathrm{C}$ for $1 \mathrm{~min}$. RNU6 was used as internal control. Relative expressions were calculated by the $2^{-\triangle \triangle \mathrm{CT}}$ method. All reactions were performed in triplicate.

\section{Luciferase assay}

Luciferase reporter assay was done by transient co-transfection of pMIR-REPORT vector (Ambion) containing wild type or mutant $3^{\prime} \mathrm{UTR}$ of human MCT1 containing the putative miR-124 binding site and miR-124 mimic or control mimic into $293 \mathrm{~T}$ cells in 24-well plates using Lipofectamine 2000 (Invitrogen) according to the manufacturer's instructions. For luciferase reporter assay, $48 \mathrm{~h}$ after transfection, luciferase activity was measured using the Dual-Luciferase Reporter Assay System (Promega). Luciferase activity was read by SpectraMax M5 (Molecular Devices, Sunnyvale, CA). Experiments were performed in triplicate and repeated three times.

\section{Western blot analysis}

Cells were lysed using RIPA buffer (\#89900, ThermoFisher, Waltham, MA, USA) with $1 \times$ protease inhibitor mix (GE Healthcare, Piscataway, NJ, USA) and incubated on ice for $15 \mathrm{~min}$. After centrifugation at $14,000 \mathrm{~g}$, the supernatant was saved. Protein concentration was determined by the Bradford assay. Equal amount of proteins was mixed with $2 \times$ sample buffer (Bio-Rad), followed by heating for $3 \mathrm{~min}$. Samples were resolved by electrophoresis were transferred to PVDF membrane and blocked with 5\% BSA for $1 \mathrm{~h}$ at room temperature. Membranes were then probed with primary antibodies at $1: 1000$ concentrations at $4{ }^{\circ} \mathrm{C}$ for overnight. After washing, membranes were incubated with secondary 
antibodies at room temperatures for $1 \mathrm{~h}$. Protein expression was detected by ECL Plus Western blotting reagents (GE Healthcare) with appropriate horseradish peroxidase-conjugated secondary antibody. Images were taken using LAS-3000 Imaging System from Fuji. All experiments were repeated three times.

\section{Xenograft experiments}

Animal experiments in this study were approved by the Affiliated Shengjing Hospital of China Medical University, China. A total of 32 female BALB/c nude mice were used. Xenograft tumors were established via injection of $1 \times 10^{6}$ MCF7 Taxol resistant cells into the fat pad of mammary gland of 6-week old mice. After tumors reached $5 \mathrm{~mm}$ in diameter, the tumor-bearing mice were randomly assigned to four groups: Control (saline, $\mathrm{n}=8$ ), Taxol alone $(n=8)$, Taxol plus SR13800 (MCT1 inhibitor) $(\mathrm{n}=8)$ and Taxol plus miR-124 mimic $(\mathrm{n}=8)$. Tumor diameters were measured with calipers twice per week for 4 weeks. The tumor volumes were calculated with the following formula: volume $\left(\mathrm{mm}^{3}=\right.$ width $\times$ length $\left./ 2\right)$, where width and length are the minor and major diameters, respectively.

\section{Statistical analysis}

Statistical analysis was performed using GraphPad Prism 5.0 software. Statistical significance between two groups was analyzed by Two-tailed Student's t-test and significance among three or more groups was analyzed by ANOVA followed by post hoc analysis. $p$ values $<0.05$ were considered significant.

\section{Results}

Taxol treatments induce lactate secretion and expressions of MCT1 and LDHA

It was known that Taxol sensitivity of cancers was tightly correlated with cellular metabolism [24]. Thus, we started to assess the effects of Taxol treatments on the intracellular lactate production and extracellular lactate secretion, both are indicators of altered glucose metabolism. Human breast cancer cell lines, BT474, SKBR3 and MCF7 were treated with variant concentrations of Taxol for $48 \mathrm{~h}$, since they have different sensitivities for Taxol (the IC50 was different). As we expected, the LDHA and MCT-1 protein expressions (Fig. 1a) and mRNA expressions (Fig. 1b-d) were significantly upregulated. Moreover, the extracellular lactate amounts were increased under Taxol treatments (Fig. 1e-g), suggesting Taxol treatments induced lactate production and secretion in breast cancer cells.
Taxol resistant breast cancer cells show upregulated glycolysis, MCT1 and LDHA expressions

To further investigate the correlation between Taxol and lactate production/secretion, we established Taxol resistant breast cancer cell line originating from MCF7 parental cells by selecting survival cells under gradually elevated Taxol treatments. Results in Fig. 1h demonstrated Taxol resistant MCF7 cells could tolerant higher concentrations of Taxol compared with their parental cells. The IC50 of Taxol resistant cells was increased to 235.4 nM, which is approximately six-fold of that from parental cells (38.5 nM). Consequently, we compared the protein and mRNA expressions of MCT1 and LDHA from Taxol parental and resistant cells. We found both MCT1 and LDHA were significantly upregulated in Taxol resistant cells (Fig. $1 \mathrm{i}-\mathrm{k}$ ). To evaluate the glycolysis rates between MCF7 parental and Taxol resistant cells, the glucose consumption and lactate production were measured. Expectedly, glucose consumption lactate product assays consistently increased in Taxol resistant cells (Fig. 1l-m). In general, promoted glycolysis is positively correlated with Taxol resistance.

\section{Inhibition of MCT1 derails lactate homeostasis and glycolysis of Taxol resistant cells}

We next evaluated whether blocking lactate export through inhibiting MCT1 could impair homeostasis of glucose metabolism of breast cancer cells. To achieve this, we treated MCF7 cells with SR13800, a cell-permeable inhibitor of MCT1 [12]. Expectedly, glucose consumption and lactate production were significantly suppressed by SR13800 treatments (Fig. 2a, b). Interestingly, we found the oxygen consumption rate was increased by blocking MCT1 (Fig. 2c), suggesting MCT1 inhibition triggered an invert "Warburg effect". In addition, the intracellular ATP/ADP ratio was significantly decreased under MCT1 inhibition (Fig. 2d). The intracellular lactate levels were increased at 8 and $16 \mathrm{~h}$ under SR13800 treatments (Fig. 2e), while the lactate export were decreased accordingly by blocking MCT1 (Fig. 2f), demonstrating a dysregulated lactate cellular accumulation under MCT1 inhibition. To further evaluate the glycolysis inhibitory effects by MCT1 inhibition, we knocked down endogenous MCT1 expressions in MCF7 cells. Consistent results showed knockdown MCT1 significantly suppressed glucose consumption (Fig. 2g) and lactate production (Fig. 2h), increased OCR (Fig. 2i) and decreased cellular ATP (Fig. 2j). Moreover, MCF7 cells with MCT1 knockdown displayed increased cellular lactate accumulation (Fig. 2k, l). Taken together, the above results demonstrated blocking MCT1 impaired lactate homeostasis of breast cancer cells. 


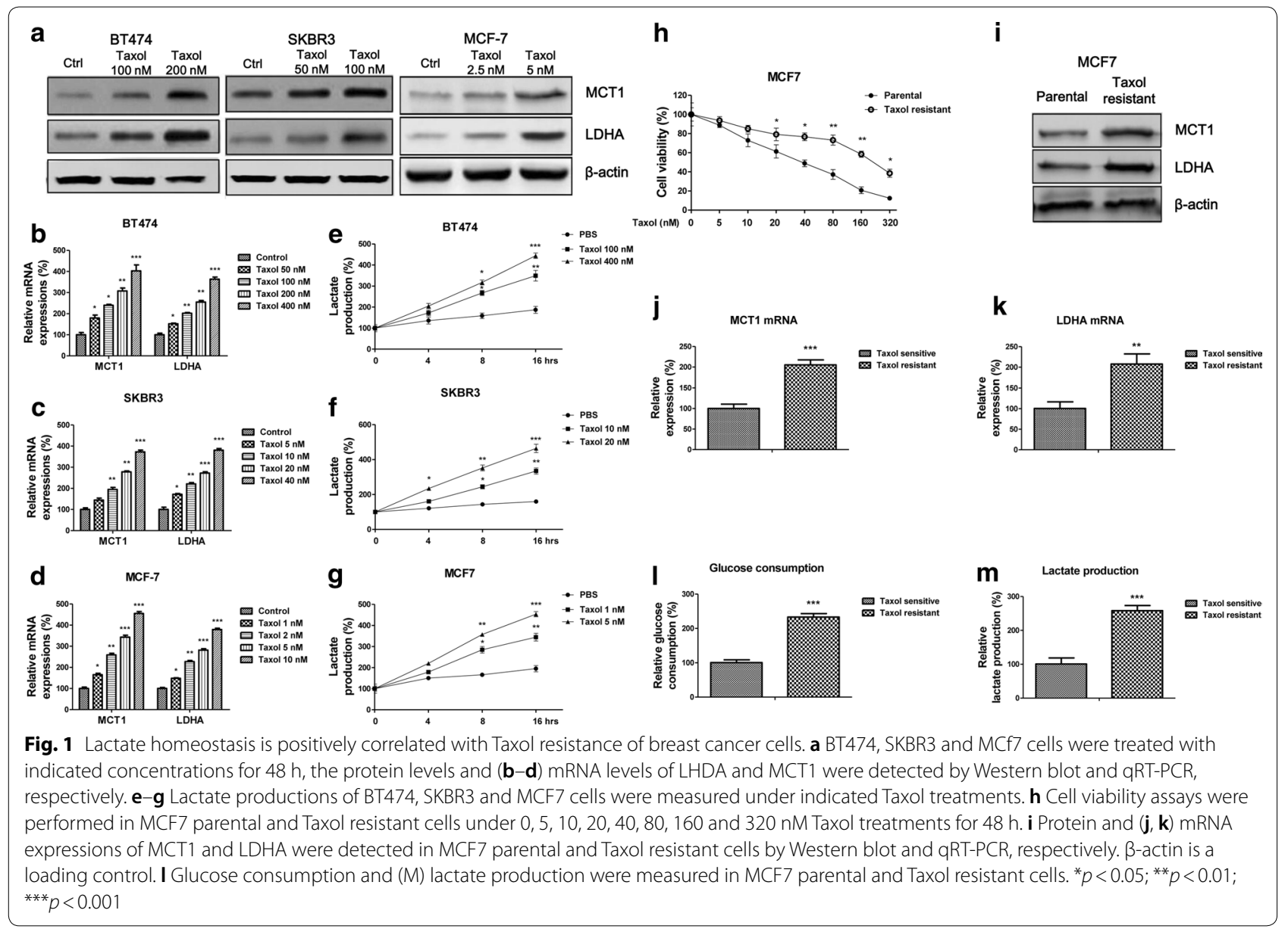

\section{MiR-124 associates with Taxol sensitivity of breast cancer cells and directly targets MCT1}

To investigate the mechanisms of the Taxol-mediated lactate homeostasis disorder, we focused on microRNAs which have been known as essentially endogenous regulators of cancer glucose metabolism. We found miR-124 expressions were significantly downregulated by treating with low concentration-Taxol in three breast cancer cells, BT474, SKBR3 and MCF7 (Fig. 3a-c), suggesting miR-124 might involve in the negative feedback of TaxolMCT1 pathway since miRNAs negatively regulate the expression of their target genes through direct binding to $3^{\prime}$ UTR region. Consistent with previous study that miR-124 acts as a tumor suppressive miRNA [16-21], we found miR-124 was downregulated in human breast cancer patient samples compared with their adjacent normal tissues (Fig. 3d). Overexpression of miR-124 suppressed cell growth rates of MCF7 cells (Fig. 3e). To investigate the roles of miR-124 in Taxol sensitivity, we compared the expressions of miR-124 in Taxol resistant cells and parental cells. As we expected, as a potential tumor suppressive miRNA, miR-124 was significantly downregulated in Taxol resistant cells (Fig. 3g). To identify potential targets of miR-124 in breast cancer cells, we searched two online miRNA target prediction algorithms, microRNA. org and TargetScan. Both software predicted MCT1 as a putative target of miR-124. MCT1 contains a conserved 7-mer nucleotide at positions $277-283$ on its $3^{\prime} U T R$ as a miR-124 binding sites (Fig. 4a). To verify whether miR124 could target MCT1 in breast cancer cells, we overexpressed miR-124 by transfecting miR-124 precursor into two breast cancer cells. MCF7 and SKBR3 cells displayed significantly downregulated MCT1 expressions (Fig. 4b). To determine whether MCT1 is a direct target of miR124 through binding to the predicted 3'UTR region, we performed luciferase assay. A fragment of around 300 base pairs of the MCT1 3'UTR containing miR-124 binding sequences downstream of the luciferase coding regions was inserted into luciferase reporter. The constructs containing either WT or mutant $3^{\prime} \mathrm{UTR}$ of MCT1 were co-transfected with control miR-124 or miR-124 precursor into 293T cells (Fig. 4c). As shown in Fig. 4c, luciferase activity of vector containing WT $3^{\prime} \mathrm{UTR}$ of MCT1 decreased about $50 \%$ by co-transfecting with 

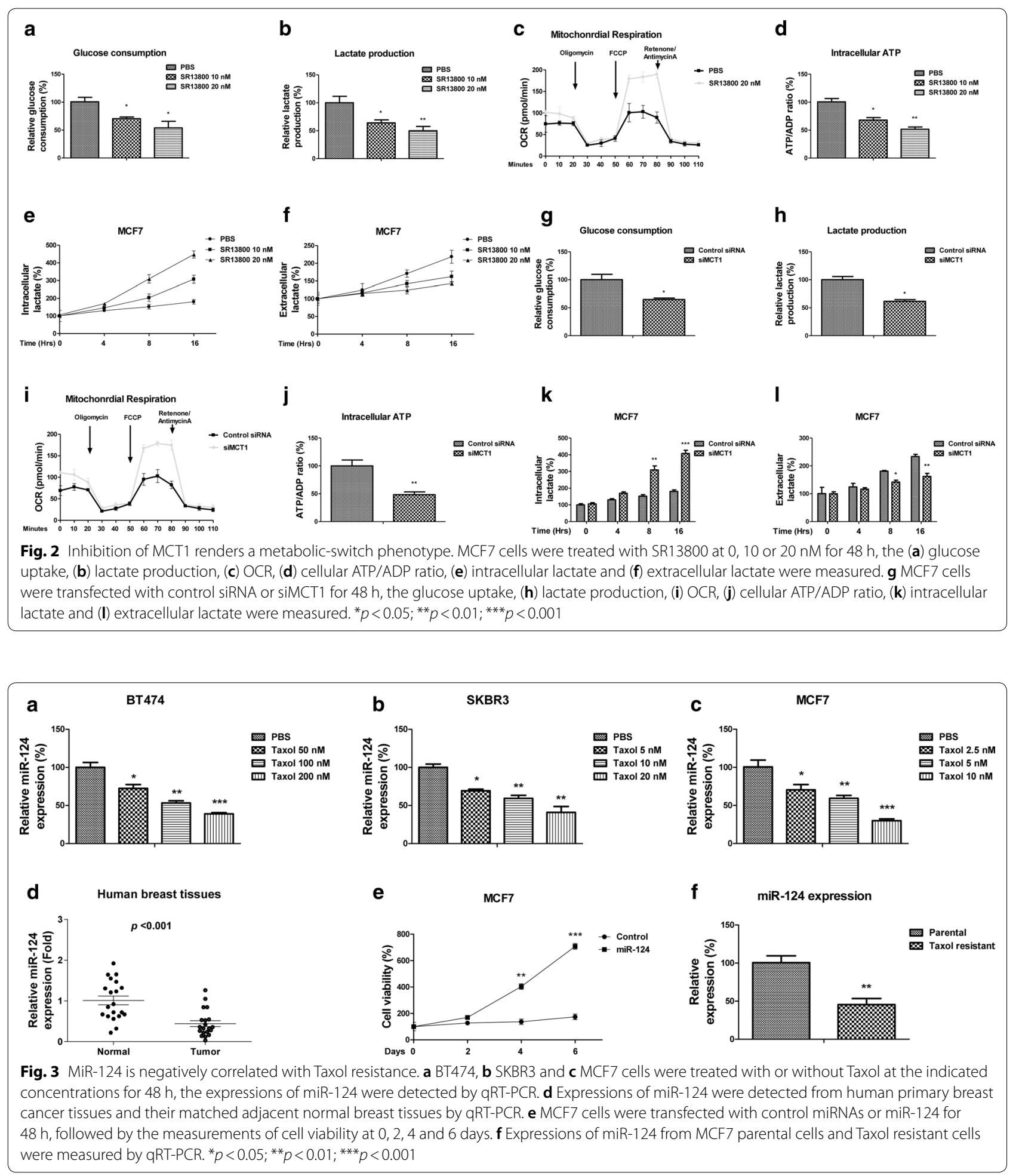

miR-124 compared with control miRNA. By contrast, the luciferase activities of vector containing mutant $3^{\prime} \mathrm{UTR}$ of MCT1 are poorly affected by miR-124 co-transfection. These data verified that miR-124 could directly target
MCT1 by binding its $3^{\prime} \mathrm{UTR}$. To investigate whether miR124 could target MCT1 in human breast cancer patients, we evaluated the correlation between MCT1 transcript levels and miR-124 expressions in a series of 60 primary 


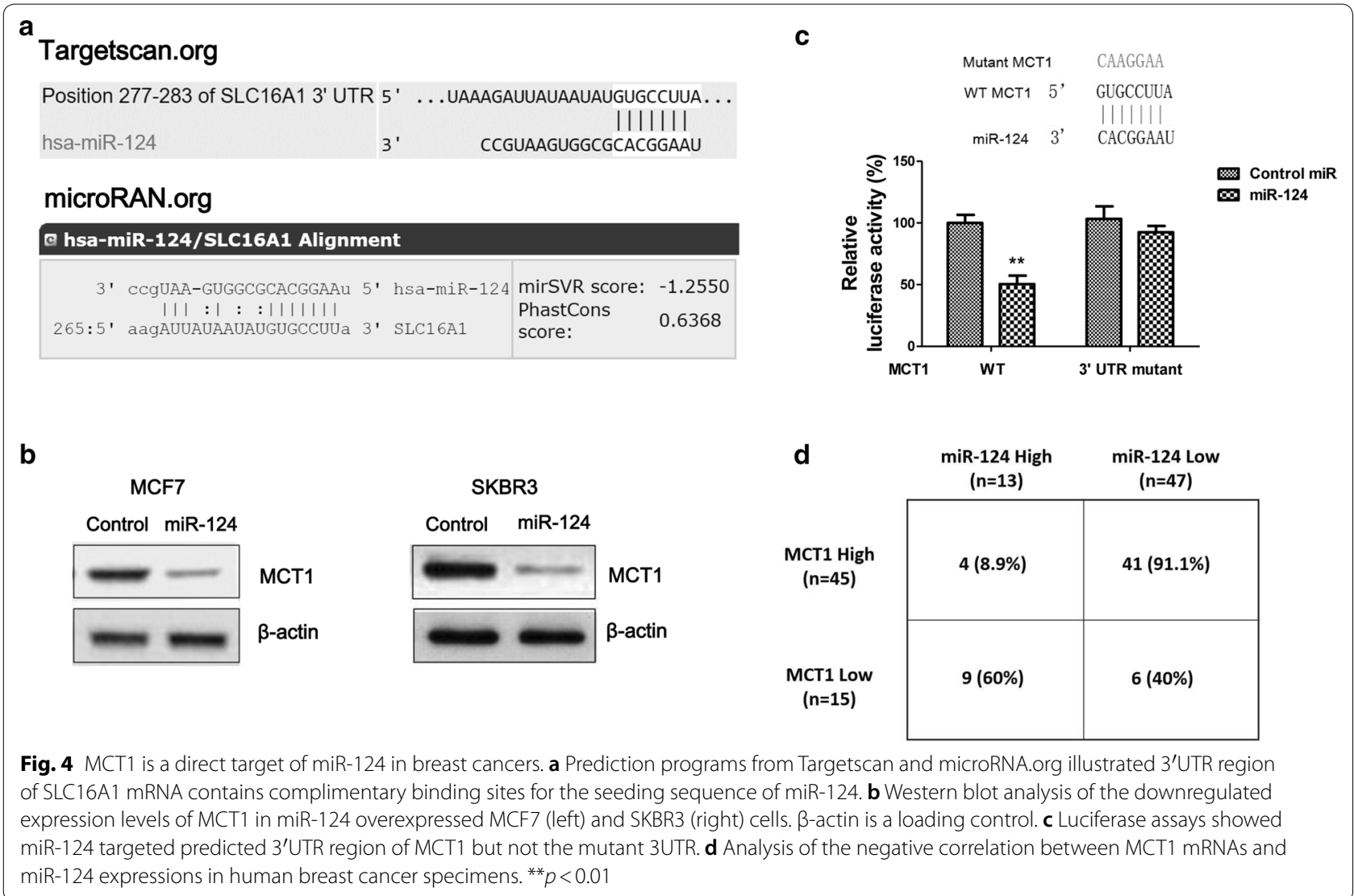

breast cancer patient samples by qRT-PCR analysis. Statistical analysis illustrated high percentages of low miR124 expression in MCT1 high-expressed samples (91.1\%), while among the MCT1 low-expressed breast cancer samples, high expressed miR-124 presented a high percentage (60\%) (Fig. 4d). Taken together, these results demonstrated miR-124 could directly target MCT1, correlating with the miR-124-mediated Taxol sensitivity.

\section{The combination of MCT1 inhibition with Taxol exhibits synergistic cytotoxicity on breast cancer cells}

Given the high correlation among miR-124, Taxol sensitivity and MCT1 in breast cancers, we wondered whether the combination of MCT1 inhibitor or miR-124 with Taxol could improve the anticancer chemotherapeutic effects. We thus treated MCF-7 and BT474 cells with control, Taxol alone, MCT1 inhibitor alone or the combination of both. Though Taxol or SR13800 alone at low concentrations had little toxic effect, co-treatment of MCF-7 or BT474 cells with Taxol and SR13800 led to significant cell death (Fig. 5a, b). Synergy of Taxol plus SR13800 was evident in these two MCT1 expressing breast cancer cells (Fig. 5a, b). Yet, as expected, no synergistic effects on MCT1 negative human breast cancer cells, MDA-MB-231 (not shown). To test whether the combination of miR-124 and Taxol could achieve synergistic inhibitory effects, we overexpressed miR-124 in MCF7 and BT474 cells (Fig. 5c, d), followed by the cotreatment of breast cancer cells with Taxol plus miR-124. As we expected, synergy of Taxol plus miR-124 was evident in MCF7 and BT474 cells (Fig. 5e, f). Taken together, the above results indicate selective targeting MCT1 could augment the anticancer potency of Taxol.

Inhibition of MCT1 by miR-124 re-sensitizes Taxol resistant cells through suppression of glycolysis in vitro and in vivo We demonstrated the negative correlation between miR124 expression and Taxol sensitivity in breast cancer cells (Fig. 3). To assess whether overexpression of miR124 could sensitize Taxol resistant cells, we co-treated MCF7 Taxol resistant cells with Taxol and MCT1 inhibitor. Results in Fig. 6a demonstrated Taxol resistant cells displayed significant cell death rate under the Taxol (10, 20, 40, 60 and $80 \mathrm{nM}$ ) plus SR13800 (5 or $10 \mathrm{nM}$ ). Similar results were observed in MCF7 Taxol resistant cell with overexpression of miR-124 (Fig. 6b), suggesting targeting MCT1 maybe an effect approach against Taxol resistance. Furthermore, Taxol resistant cells had decreased 


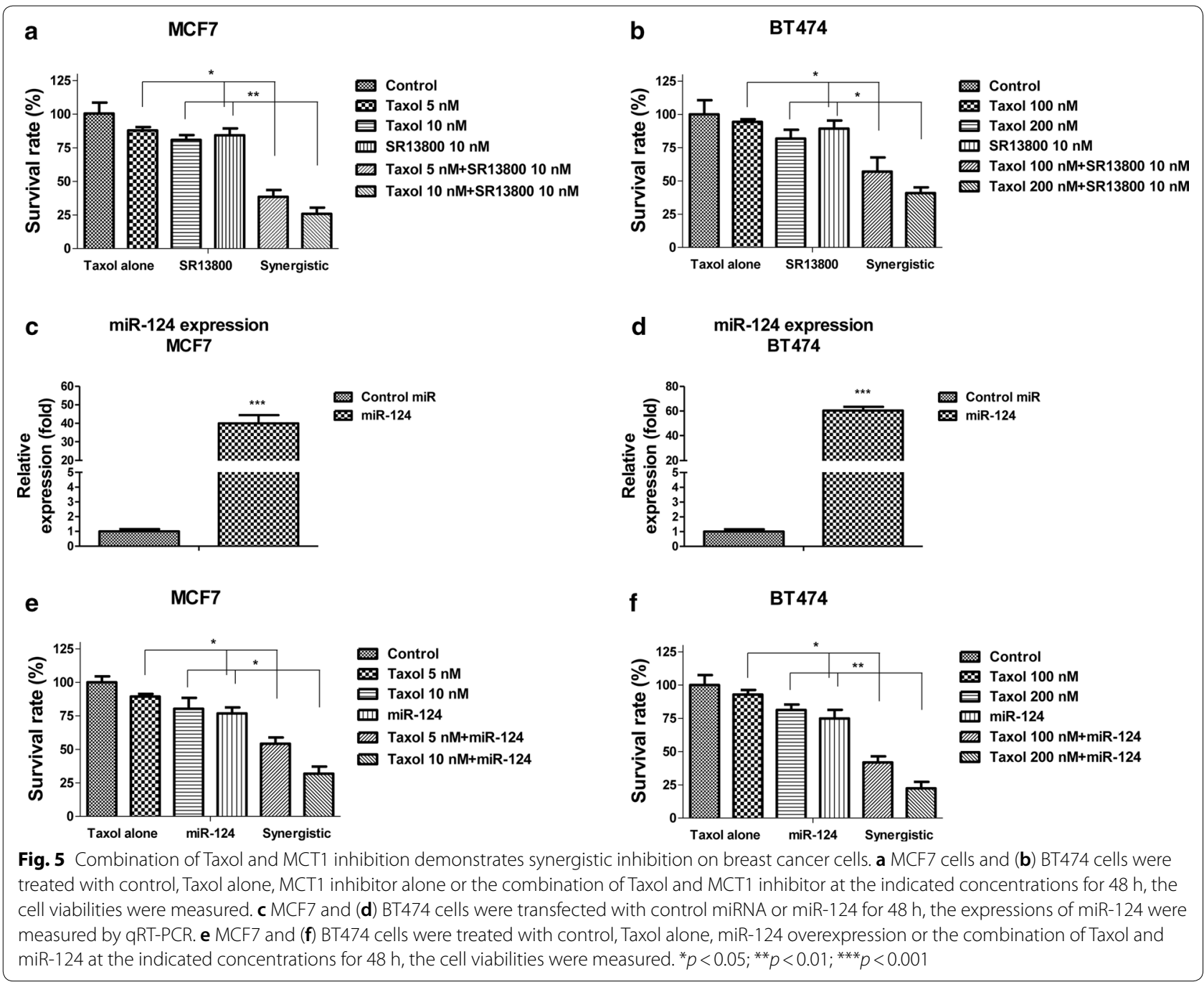

levels of glucose consumption (Fig. 6c) and intracellular ATP (Fig. 6d), increased intracellular lactate accumulation (Fig. 6e) and decreased lactate exportation (Fig. 6f) under either overexpression of miR-124 or MCT1 inhibitor treatments. These findings present a strong correlation between Taxol sensitivity and the MCT1-mediated lactate secretion. To verify whether the miR-124-modulated Taxol sensitivity was through MCT1 inhibition, we performed rescue experiments by endogenously overexpression of MCT1 into miR-124-overexpressing Taxol resistant cells (Fig. 6g). Notably, restoration of MCT1 significantly promoted the survival rates of miR-124-overexpressing Taxol resistant cells under $20,40,80$ or $180 \mathrm{nM}$ Taxol treatments (Fig. 6h). Therefore, in vitro rescue experiments demonstrated sensitization of Taxol resistant cells by miR-124 was through inhibiting MCT1. To examine whether inhibiting MCT1 impacts Taxol sensitivity of breast cancer tumors in vivo, we performed xenograft experiments by injection of MCF7 Taxol resistant cells into nude mice and began Taxol alone, Taxol plus MCT1 inhibitor or Taxol plus miR-124 mimic treatment after the tumors reached $5 \mathrm{~mm}$ in diameter. The combination of Taxol with either SR13800 or miR-124 mimic robustly suppressed growth of the mammary fat pad xenograft tumors (Fig. 7a). The tumor sizes of the combined treatments were significantly smaller than control or Taxol alone treatment (Fig. 7b, c). Moreover, qPCR results illustrated the MCT1 mRNA levels were significantly downregulated by miR-124 mimic or SR 13800 treatments compared with control or Taxol alone treatment (Fig. 7d). These data are consistent with our in vitro results showing that miR-124 sensitizes Taxol resistant breast cancer cells through MCT1 inhibition.

\section{Discussion}

It was known that increased aerobic glycolysis is a hallmark of cancer cells [6]. Glucose consumption and glycolytic flux is augmented, leading to the production of 


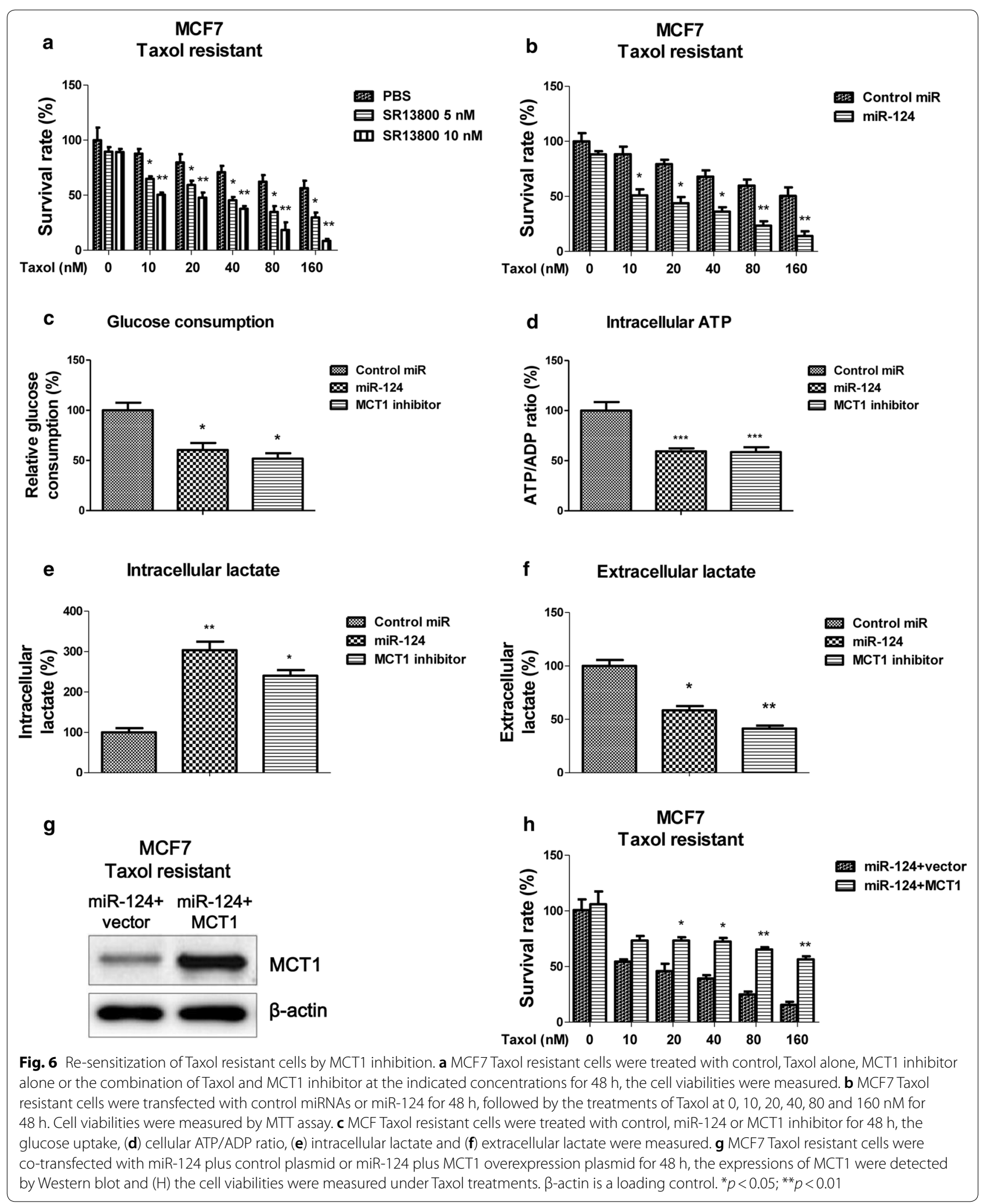



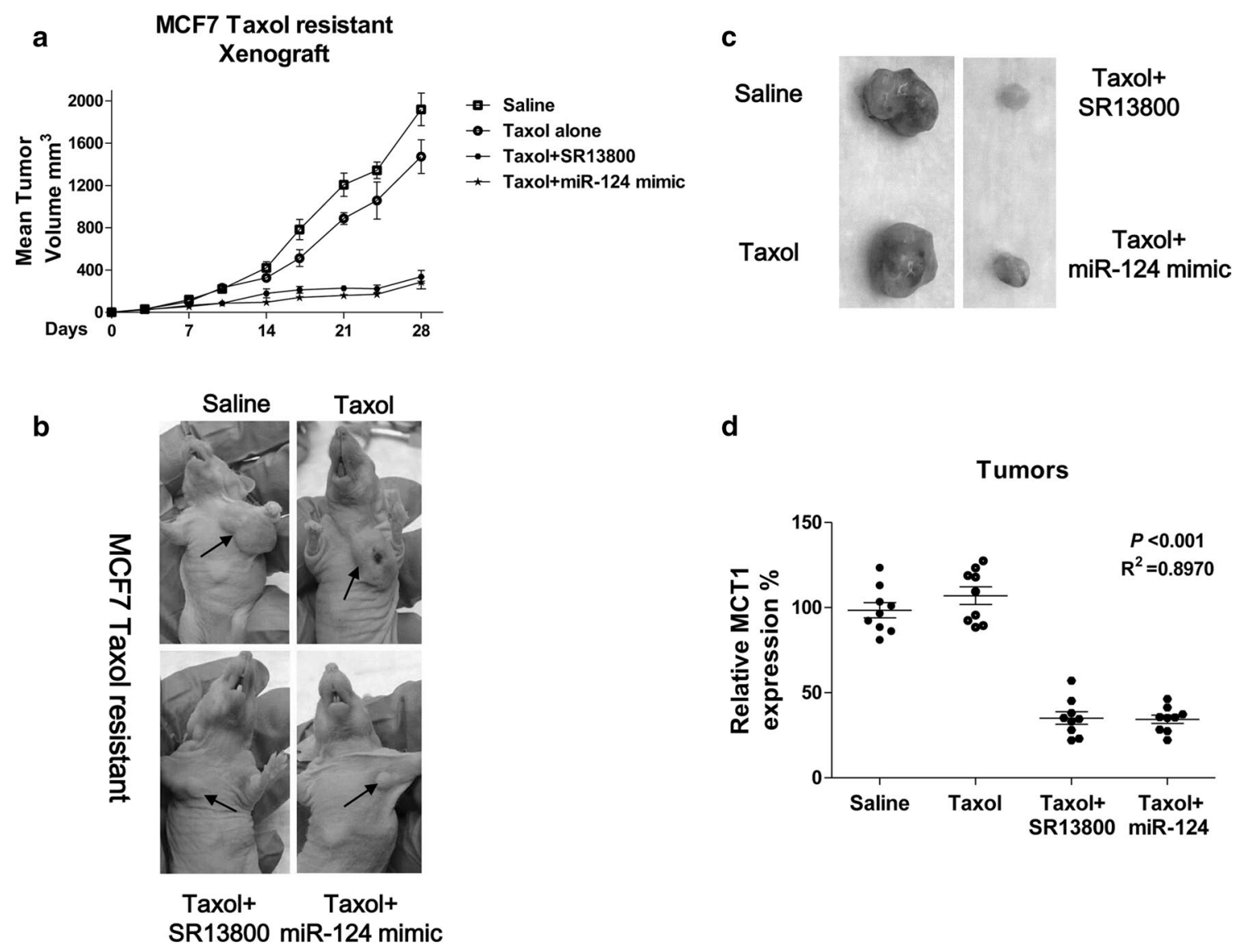

d

Tumors

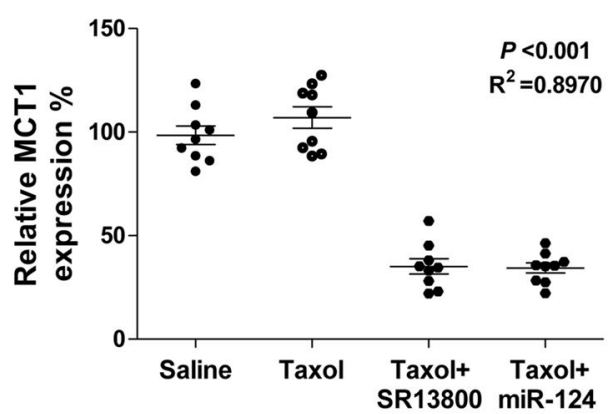

Fig. 7 Combination of Taxol and MCT1 inhibition effectively suppresses Taxol resistant tumor growth in vivo. a-c Comparison of tumor volumes in pre-established MCF7 Taxol resistant cells tumor xenografts under treatments with control, Taxol alone (10 mg/kg, i.p., 3 times/wk), Taxol plus SR13800 (10 mg/kg, i.p., 3 times/wk), or Taxol plus miR-124 mimic (5 $\mu \mathrm{L}$ of $50 \mu \mathrm{M}$, i.p., 3 times/wk). d MCT1 mRNA expressions in MCF7 Taxol resistant cells tumor xenograft treated with control, Taxol alone, Taxol plus SR13800 and Taxol plus miR-124 mimic. ${ }^{*} p<0.05 ;{ }^{* *} p<0.01$

intermediates necessary for fast growth of cancer cells [6]. Furthermore, mounting evidence attributes the chemoresistance to dysregulated cellular metabolism [25]. In this study, we demonstrated low dose Taxol treatments induced glycolysis flux of breast cancer cells. Consequently, Taxol resistant cells displayed upregulated glucose consumption and lactate production, suggesting targeting glycolysis may result in augments of chemotherapeutic effects.

Under stress, metabolic reprogramming provides cancer cells survival advantage, presenting one of the hallmarks of cancer. Recent studies and our results demonstrated MCT1 is upregulated in human malignancies [11], suggesting an oncogenic role of MCT1. Our findings showed that inhibition of MCT1 by either specific siRNA knockdown or inhibitor significantly suppressed glycolysis and lactate homeostasis of breast cancer cells. In addition, we report inhibition of MCT1 led to a metabolic shift from anaerobic glycolysis to oxidative phosphorylation, a revered "Warburg effects". These findings are consistent with previous studies showing another MCT1 inhibitor, AZD3965 could reduce glycolysis and proliferation rates but increases oxygen consumption [26]. In addition to their discoveries, we firstly demonstrated MCT1 inhibition by either siRNA knockdown or inhibitor treatment sensitizes Taxol resistant breast cancer cell. In our mammary fat pad xenograft model, Taxol plus MCT1 inhibition treatment resensitized Taxol resistant tumor growth. Our in vitro and in vivo results consistently showed synergistic antitumor effects with the combined treatments of Taxol and MCT1 inhibition, providing new approach for anti-chemoresistance of breast cancer. However, the mechanism by which blocking MCT1 impacts Taxol sensitivity remains unclear. We showed inhibition of MCT1 led to accumulation of intracellular lactate, which may trigger endogenous toxicity of breast cancer cells. These results are consistent with previous study [12]. In addition, regulation of cellular redox status, disruption of intracellular $\mathrm{pH}$ homeostasis, or bioenergetics pathway could be possible mechanisms. 
Studies indicate that miR-124 plays a tumor suppressive role in malignant progression of human cancers [16-21]. It has been reported that miR-124 inhibits proliferation in cancer cells through targeting CDK4 [21], CBL [27], Slug [28] and androgen receptor [29]. We firstly demonstrated MCT1 as a direct target of miR-124 in breast cancer cells and patient specimens. MiR-124 directly binds the 3'-UTR of MCT1. Notably, miR-124 was downregulated in Taxol resistant cells compared with parental cells, consistent with previous description that miR-124 has tumor suppressive functions. Furthermore, rescue experiments supported overexpression of miR-124 suppressed proliferation rates and increased Taxol sensitivity of breast cancer cells through targeting MCT1, indicating miR-124 may serve as an ideal therapeutic target against chemoresistant breast cancer.

\section{Conclusion}

In summary, our study reveals a miR-124-MCT1-Taxol sensitivity axis, providing a new mechanism for the miRNA-mediated Taxol sensitivity. These findings may provide insight into the identification of novel therapeutic targets against chemoresistant breast cancers.

\begin{abstract}
Abbreviations
MCT1: monocarboxylate transporter 1; LDHA: lactate dehydrogenase-A; miRNAs: microRNAs; UTR: untranslated region; OSCC: oral squamous cell carcinoma; HCC: hepatocellular carcinoma; DMSO: dimethyl sulfoxide; IACUC: Institute Animal Care and Use Committee.
\end{abstract}

\section{Acknowledgements}

The study was supported by Project of Liaoning Natural Science Foundation (No. 2015020544).

\section{Authors' contributions \\ YZ conceived, designed and supervised the study, and participated in data acquisition, analysis and interpretation. LH, GS, YM provided material for the study, participated in pathology assessments, provided technical support, and participated in data analysis and interpretation. XJ, SJ, YF and YC provided material for the study and participated in data acquisition. LH, GS, YM and XJ participated in data acquisition. LH and GS participated in data acquisition, analysis, and interpretation. All authors were involved in manuscript writing and reviewing, gave their final approval and agreed to be accountable for all aspects of the work. All authors read and approved the final manuscript.}

\section{Funding}

The study was supported by Project of Liaoning Natural Science Foundation (No. 2015020544).

Availability of data and materials
Please contact author for data requests

\section{Ethics approval and consent to participate}

This research has been conducted in accordance with the Declaration of Helsinki. The study protocol has been approved by the Ethics Committee of the Affiliated Shengjing Hospital of China Medical University. Patients signed the informed consent form.

\section{Consent for publication}

All listed authors have actively participated in the study and have read and approved the submitted manuscript.

\section{Competing interests}

The authors declare that they have no competing interests.

Received: 25 March 2019 Accepted: 10 July 2019

Published online: 24 July 2019

\section{References}

1. Libson S, Lippman M. A review of clinical aspects of breast cancer. Int Rev Psychiatry. 2014;26(1):4-15.

2. Yeo B, Turner NC, Jones A. An update on the medical management of breast cancer. BMJ. 2014;348:g3608.

3. Sun J, Chia S. Adjuvant chemotherapy and HER-2-directed therapy for early-stage breast cancer in the elderly. Br J Cancer. 2017;1 16(1):4-9.

4. Weaver BA. How Taxol/paclitaxel kills cancer cells. Mol Biol Cell. 2014;25(18):2677-81.

5. Barbuti AM, Chen ZS, Wang H, Vo T, Hajar A, Li S, Chen X, Parissenti AM, Brindley DN, Wang Z. Multiple mechanisms underlying acquired resistance to taxanes in selected docetaxel-resistant MCF-7 breast cancer cells. BMC Cancer. 2014;14(1):37.

6. Liberti MV, Locasale JW. The warburg effect: how does it benefit cancer cells? Trends Biochem Sci. 2016;41(3):211-8.

7. Allison SJ, Knight JR, Granchi C, Rani R, Minutolo F, Milner J, Phillips RM. Identification of LDH-A as a therapeutic target for cancer cell killing via (i) p53/NAD(H)-dependent and (ii) p53-independent pathways. Oncogenesis. 2014;3(5):e102.

8. Warburg O. On the origin of cancer cells. Science. 1956;123(3191):309-14.

9. Halestrap AP, Price NT. The proton-linked monocarboxylate transporter (MCT) family: structure, function and regulation. Biochem J. 1999;343Pt2(2):281-99.

10. Pérez-Escuredo J, Van Hée VF, Sboarina M, Falces J, Payen VL, Pellerin L, Sonveaux P. Monocarboxylate transporters in the brain and in cancer. Biochim Biophys Acta. 2016;1863(10):2481-97.

11. Johnson JM, Cotzia P, Fratamico R, Mikkilineni L, Chen J, Colombo D, Mollaee M, Whitaker-Menezes D, Domingo-Vidal M, Lin Z, et al. MCT1 in invasive ductal carcinoma: monocarboxylate metabolism and aggressive breast Cancer. Front Cell Dev Biol. 2017;5:27.

12. Doherty JR, Yang C, Scott KE, Cameron MD, Fallahi M, Li W, Hall MA, Amelio AL, Mishra JK, Li F, et al. Blocking lactate export by inhibiting the Myc target MCT1 disables glycolysis and glutathione synthesis. Cancer Res. 2014;74(3):908-20.

13. Peng $\mathrm{Y}$, Croce $\mathrm{CM}$. The role of MicroRNAs in human cancer. Signal Transduct Target Ther. 2016;1:15004.

14. Rupaimoole R, Slack FJ. MicroRNA therapeutics: towards a new era for the management of cancer and other diseases. Nat Rev Drug Discov. 2017;16(3):203-22.

15. Gulyaeva LF, Kushlinskiy NE. Regulatory mechanisms of microRNA expression. J Transl Med. 2016;14(1):143.

16. Hunt S, Jones AV, Hinsley EE, Whawell SA, Lambert DW. MicroRNA-124 suppresses oral squamous cell carcinoma motility by targeting ITGB1. FEBS Lett. 2011;585(1):187-92.

17. Fowler A, Thomson D, Giles K, Maleki S, Mreich E, Wheeler H, Leedman P, Biggs M, Cook R, Little N, et al. miR-124a is frequently down-regulated in glioblastoma and is involved in migration and invasion. Eur J Cancer. 2011;47(6):953-63.

18. Chen Z, Liu S, Tian L, Wu M, Ai F, Tang W, Zhao L, Ding J, Zhang L, Tang A. miR-124 and miR-506 inhibit colorectal cancer progression by targeting DNMT3B and DNMT1. Oncotarget. 2015;6(35):38139-50.

19. Zhao X, Lu C, Chu W, Zhang B, Zhen Q, Wang R, Zhang Y, Li Z, Lv B, Li H, Liu J. MicroRNA-124 suppresses proliferation and glycolysis in nonsmall cell lung cancer cells by targeting AKT-GLUT1/HKII. Tumour Biol. 2017;39(5):1010428317706215.

20. Cai QQ, Dong YW, Wang R, Qi B, Guo JX, Pan J, Liu YY, Zhang CY, Wu XZ. MiR-124 inhibits the migration and invasion of human hepatocellular carcinoma cells by suppressing integrin aV expression. Sci Rep. 2017;7:40733.

21. Zhang T, Wang J, Zhai X, Li H, Li C, Chang J. MiR-124 retards bladder cancer growth by directly targeting CDK4. Acta Biochim Biophys Sin. 2014;46(12):1072-9. 
22. Ye M, Qiu H, Cao Y, Zhang M, Mi Y, Yu J, Wang C. Curcumin improves palmitate-induced insulin resistance in human umbilical vein endothelial cells by maintaining proteostasis in endoplasmic reticulum. Front Pharmacol. 2017;8:148.

23. Ohayon D, De Chiara A, Chapuis N, Candalh C, Mocek J, Ribeil JA, Haddaoui L, Ifrah N, Hermine O, Bouillaud F, et al. Cytoplasmic proliferating cell nuclear antigen connects glycolysis and cell survival in acute myeloid leukemia. Sci Rep. 2016;6:35561.

24. Li W, Liu J, Jackson K, Shi R, Zhao Y. Sensitizing the therapeutic efficacy of taxol with shikonin in human breast cancer cells. PLoS ONE. 2014;9(4):e94079.

25. Zhao Y, Butler EB, Tan M. Targeting cellular metabolism to improve cancer therapeutics. Cell Death Dis. 2013;4(3):e532.

26. Hong CS, Graham NA, Gu W, Espindola Camacho C, Mah V, Maresh EL, Alavi M, Bagryanova L, Krotee PAL, Gardner BK, et al. MCT1 Modulates Cancer Cell Pyruvate Export and Growth of Tumors that Co-express MCT1 and MCT4. Cell Rep. 2016;14(7):1590-601.
27. Wang Y, Chen L, Wu Z, Wang M, Jin F, Wang N, Hu X, Liu Z, Zhang CY, Zen $\mathrm{K}$, et al. miR-124-3p functions as a tumor suppressor in breast cancer by targeting CBL. BMC Cancer. 2016;16(1):826.

28. Liang YJ, Wang QY, Zhou CX, Yin QQ, He M, Yu XT, Cao DX, Chen GQ, He JR, Zhao Q. MiR-124 targets Slug to regulate epithelial-mesenchymal transition and metastasis of breast cancer. Carcinogenesis. 2013;34(3):713-22.

29. Shi XB, Xue L, Ma AH, Tepper CG, Gandour-Edwards R, Kung HJ, deVere White RW. Tumor suppressive miR-124 targets androgen receptor and inhibits proliferation of prostate cancer cells. Oncogene. 2013;32(35):4130-8.

\section{Publisher's Note}

Springer Nature remains neutral with regard to jurisdictional claims in published maps and institutional affiliations.
Ready to submit your research? Choose BMC and benefit from:

- fast, convenient online submission

- thorough peer review by experienced researchers in your field

- rapid publication on acceptance

- support for research data, including large and complex data types

- gold Open Access which fosters wider collaboration and increased citations

- maximum visibility for your research: over $100 \mathrm{M}$ website views per year

At BMC, research is always in progress.

Learn more biomedcentral.com/submissions 